\title{
Shape Sensitivity Analysis of the Dirichlet Laplacian in a Half-Space
}

by

\author{
Cherif AMROUCHE, Šárka NEČASOVÁ and Jan SOKOŁOWSKI
}

\author{
Presented by Bogdan BOJARSKI
}

\begin{abstract}
Summary. Material and shape derivatives for solutions to the Dirichlet Laplacian in a half-space are derived by an application of the speed method. The proposed method is general and can be used for shape sensitivity analysis in unbounded domains for the Neumann Laplacian as well as for the elasticity boundary value problems.
\end{abstract}

1. Introduction. In the paper we combine the recent results on boundary value problems in unbounded domains [1], [3], [2] with the speed method of shape sensitivity analysis [8], [4], in order to define the material and shape derivatives of solutions. In the case of bounded domains the related results can be found in [8]. The case of exterior domains is also already treated in the habilitation thesis of J.-P. Zolésio (1979). However, to the best of our knowledge, the case of unbounded domains has not been considered in the literature on shape optimization.

1.1. Shape sensitivity analysis. There are numerous applications of shape sensitivity analysis to numerical solution of shape optimization problems, inverse problems, and topological sensitivity analysis of shape functionals. We refer the reader, e.g., to [8] for an introduction to the subject in bounded domains, and to [4] for recent results on evolution of geometrical domains.

From the point of view of shape sensitivity analysis there is a substantial difference between the so-called exterior problems and boundary value problems (BVP) in unbounded domains. Therefore, we describe in detail the mathematical framework for a model problem in a half-space, which is used

2000 Mathematics Subject Classification: 35J05, 49Q10, 74P99.

Key words and phrases: shape derivative, material derivative, speed method, Dirichlet Laplacian. 
to characterize the dependence of solutions to an elliptic equation with respect to boundary variations. As a result, the form of material derivatives, shape derivatives and shape gradients of integral functionals are given for the Dirichlet Laplacian in a half-space.

The same method can be used for the Neumann Laplacian, and systems of equations in unbounded domains.

1.2. Dirichlet Laplacian in variable domains-formal definition. First we describe the problem of shape sensitivity analysis in a formal way and after introducing appropriate spaces we present the precise formulation of the problem.

We consider the shape sensitivity analysis of the model problem

$$
\begin{array}{ll}
-\Delta u=f & \text { in } \Omega, \\
u=g & \text { on } \Gamma,
\end{array}
$$

where $\Omega=\mathbb{R}_{+}^{N}$ and $\Gamma=\partial \Omega=\mathbb{R}^{N-1}$. Following [8] we introduce the mapping $T_{t}: \mathbb{R}^{N} \rightarrow \mathbb{R}^{N}$ associated with the velocity field $V(t, x)$ which is compactly supported with respect to the spatial variable $x$. The mapping is given by the system of differential equations

$$
\begin{aligned}
& \frac{d}{d t} x(t)=V(t, x(t)), \\
& x(0)=X,
\end{aligned}
$$

with the solution denoted by $x(t)=x(t, X), t \in(-\delta, \delta), X \in \mathbb{R}^{N}$. The variable domain $\Omega_{t}=T_{t}(\Omega)$ is defined in the usual way, $\Omega_{t}=\left\{x \in \mathbb{R}^{N}\right.$ | $x=x(t, X), X \in \Omega\}$. Therefore, we can consider the Laplace equation in $\Omega_{t}$,

$$
\begin{array}{ll}
-\Delta u_{t}=f_{t} & \text { in } \Omega_{t}=T_{t}\left(\mathbb{R}_{+}^{N}\right), \\
u_{t}=g_{t} & \text { on } \Gamma_{t}=T_{t}\left(\mathbb{R}^{N-1}\right) .
\end{array}
$$

The solution transported to the fixed domain is denoted by $u^{t}=u_{t} \circ T_{t}$, and formally we can write [8] that it satisfies the following equations:

$$
\begin{array}{ll}
-\operatorname{div}\left(A(t) \nabla u^{t}\right)=F^{t} & \text { in } \Omega, \\
u^{t}=g^{t} & \text { on } \partial \Omega,
\end{array}
$$

where we use the same notation as in [8]:

$$
\begin{aligned}
A(t) & =\operatorname{det}\left(D T_{t}\right)\left(D T_{t}^{-1}\right)^{*} D T_{t}^{-1}, \\
F^{t} & =\operatorname{det}\left(D T_{t}\right)\left(f_{t} \circ T_{t}\right), \\
f^{t} & =f_{t} \circ T_{t}, \quad g^{t}=g_{t} \circ T_{t}, \\
\gamma(t) & =\operatorname{det}\left(D T_{t}\right),
\end{aligned}
$$

and $M^{*}$ is the transpose of $M$. 
For $t \in(-\delta, \delta)$ the matrix $A(t)$ stabilizes for $|x| \rightarrow \infty$ to the identity matrix, since the field $V$ is compactly supported. We are looking for solutions to (PP) in weighted Sobolev spaces, with weights which describe the behavior of functions at infinity.

By an application of the implicit function theorem in weighted spaces, using the estimates obtained for the model problem, we can prove the existence of the material and shape derivatives for the solution to (1.1).

The material derivative $\dot{u}$ is given by the solution of the following problem:

$$
\begin{array}{ll}
-\Delta \dot{u}=\dot{f}+\operatorname{div} V f+\operatorname{div}\left(A^{\prime}(0) \nabla u\right) & \text { in } \Omega, \\
\dot{u}=\dot{g} & \text { on } \partial \Omega,
\end{array}
$$

where $u$ is the solution of the problem (1.1). Here we define

$$
\dot{u}=\lim _{t \rightarrow 0} \frac{u^{t}-u}{t},
$$

in the norm of the energy space, $\dot{f}, \dot{g}$ are defined in the same way, and we have $\dot{f}=\nabla f \cdot V, \dot{g}=\nabla g \cdot V$, since $f_{t}, g_{t}$ are the restrictions of given functions to $\Omega_{t}$.

Since the shape gradients of differentiable shape functionals depend on the so-called shape derivative

$$
u^{\prime}=\dot{u}-\nabla u \cdot V,
$$

we also show the existence of the shape derivative $u^{\prime}$ which can be characterized as the solution to the Laplace equation

$$
\begin{array}{ll}
-\Delta u^{\prime}=0 & \text { in } \Omega, \\
u^{\prime}=-\frac{\partial u}{\partial n} V \cdot n & \text { on } \partial \Omega,
\end{array}
$$

with $n=(0, \ldots, 0,-1)^{\top}$ the exterior normal vector to $\Gamma$, so $\partial / \partial n=-\partial / \partial x_{N}$. Problem (1.6) is obtained from (1.3), taking into account relation (1.5), and for the extension of $g$ from the boundary to $\mathbb{R}^{N}$ which satisfies the condition $\partial g / \partial n=0$.

Our plan is the following: after introduction of the function spaces and preliminary lemmas we recall the known results on the Laplace equations with non-homogeneous Dirichlet conditions in the particular case of $f \in$ $\dot{W}_{1}^{0,2}\left(\mathbb{R}_{+}^{N}\right)$ (resp. $g \in W_{1}^{3 / 2,2}\left(\mathbb{R}^{N-1}\right)$ ). Then we show the existence of the material derivatives of $\dot{f}$ (resp. $\dot{g}$ ) under the assumption that the mapping $t \mapsto f \circ T_{t}$ (resp. $t \mapsto g \circ T_{t}$ ) is weakly differentiable. Further we are interested in the solutions of problem (TP) and (PP) and we prove the existence of the material derivatives for $u$. Finally, we look for $\dot{u}, u^{\prime}$ as solutions of Laplace equations and we obtain the shape gradient of the energy functional. 
We introduce a class of weighted spaces and some preliminary lemmas. From the point of view of shape sensitivity analysis, the main difference with the classical analysis in bounded domains consists in a slightly different setting for the variational formulation. Thus, we provide a detailed introduction to the functional setting for such problems.

Problem $(\mathrm{P})$ has been investigated in weighted Sobolev spaces by several authors, but only in the Hilbert space setting $(p=2)$ and without critical cases corresponding to the logarithmic factor (cf. [3], [6]).

Let $\Omega$ be an open set in $\mathbb{R}^{N}, N \geq 2$. Let $x=\left(x_{1}, \ldots, x_{N}\right)$ be a point in $\mathbb{R}^{N}$ and $r=|x|=\left(x_{1}^{2}+\cdots+x_{N}^{2}\right)^{1 / 2}$. We shall use two basic weights:

$$
\varrho=\left(1+r^{2}\right)^{1 / 2} \text { and } \lg \varrho=\ln \left(2+r^{2}\right) .
$$

As usual, $\mathcal{D}\left(\mathbb{R}^{N}\right)$ denotes the spaces of infinitely differentiable functions with compact supports and $\mathcal{D}^{\prime}\left(\mathbb{R}^{N}\right)$ denotes its dual space, called the space of distributions. For any nonnegative integers $n$ and $m$, and real numbers $p>1$, $\alpha$ and $\beta$, setting

$$
k=k(m, N, p, \alpha)= \begin{cases}-1 & \text { if } N / p+\alpha \notin\{1, \ldots, m\}, \\ m-N / p-\alpha & \text { if } N / p+\alpha \in\{1, \ldots, m\},\end{cases}
$$

we define the following space:

$$
\begin{aligned}
& W_{\alpha, \beta}^{m, p}(\Omega) \\
& =\left\{u \in \mathcal{D}^{\prime}(\Omega) \mid \varrho^{\alpha-m+|\lambda|}(\lg \varrho)^{\beta-1} D^{\lambda} u \in L^{p}(\Omega) \text { for } 0 \leq|\lambda| \leq k ;\right. \\
& \left.\varrho^{\alpha-m+|\lambda|}(\lg \varrho)^{\beta} D^{\lambda} u \in L^{p}(\Omega) \text { for } k+1 \leq|\lambda| \leq m\right\} .
\end{aligned}
$$

In the case $\beta=0$, we simply denote the above space by $W_{\alpha}^{m, p}(\Omega)$. Note that $W_{\alpha, \beta}^{m, p}(\Omega)$ is a reflexive Banach space equipped with its natural norm:

$$
\begin{aligned}
\|u\|_{W_{\alpha, \beta}^{m, p}(\Omega)}= & {\left[\sum_{0 \leq|\lambda| \leq k}\left\|\varrho^{\alpha-m+|\lambda|}(\lg \varrho)^{\beta-1} D^{\lambda} u\right\|_{L^{p}(\Omega)}^{p}\right.} \\
& \left.+\sum_{k+1 \leq|\lambda| \leq m}\left\|\varrho^{\alpha-m+|\lambda|}(\lg \varrho)^{\beta} D^{\lambda} u\right\|_{L^{p}(\Omega)}^{p}\right]^{1 / p} .
\end{aligned}
$$

We also define the seminorm

$$
|u|_{W_{\alpha, \beta}^{m, p}(\Omega)}=\left(\sum_{|\lambda|=m}\left\|\varrho^{\alpha}(\lg \varrho)^{\beta} D^{\lambda} u\right\|_{L^{p}(\Omega)}^{p}\right)^{1 / p},
$$

and for any integer $q$, we denote by $P_{q}$ the space of polynomials in $N$ variables of degree smaller than or equal to $q$, with the convention that $P_{q}$ is reduced to $\{0\}$ for negative $q$. The weights in definition (1.7) are chosen so that the corresponding space has two properties:

$$
\mathcal{D}\left(\overline{\mathbb{R}_{+}^{N}}\right) \text { is dense in } \quad W_{\alpha, \beta}^{m, p}\left(\mathbb{R}_{+}^{N}\right),
$$

and a Poincaré-type inequality holds in $W_{\alpha, \beta}^{m, p}\left(\mathbb{R}_{+}^{N}\right)$. 
THEOREM 1.1. Let $\alpha$ and $\beta$ be two real numbers and $m \geq 1$ an integer not satisfying simultaneously

$$
N / p+\alpha \in\{1, \ldots, m\} \quad \text { and } \quad(\beta-1) p=-1 .
$$

Then the seminorm $|\cdot|_{W_{\alpha, \beta}^{m, p}\left(\mathbb{R}_{+}^{N}\right)}$ defines on $W_{\alpha, \beta}^{m, p}\left(\mathbb{R}_{+}^{N}\right) / P_{q^{\prime}}$ a norm which is equivalent to the quotient norm, with $q^{\prime}=\inf (q, m-1)$, where $q$ is the highest degree of the polynomials contained in $W_{\alpha}^{m, p}\left(\mathbb{R}_{+}^{N}\right)$.

Proof. See [2].

Now, we define the space

$$
\stackrel{\circ}{W}_{\alpha, \beta}^{m, p}\left(\mathbb{R}_{+}^{N}\right)=\overline{\mathcal{D}\left(\mathbb{R}_{+}^{N}\right)}\|\cdot\|_{W_{\alpha, \beta}^{m, p}\left(\mathbb{R}_{+}^{N}\right)}
$$

and the dual space of $\stackrel{\circ}{W}_{\alpha, \beta}^{m, p}\left(\mathbb{R}_{+}^{N}\right)$ is denoted by $W_{-\alpha,-\beta}^{-m, p^{\prime}}\left(\mathbb{R}_{+}^{N}\right)$, where $p^{\prime}$ is the conjugate of $p$, i.e. $1 / p+1 / p^{\prime}=1$.

THEOREM 1.2. Assume that the assumptions of Theorem 1.1 are fullfiled. Then the seminorm $|\cdot|_{W_{\alpha, \beta}^{m, p}\left(\mathbb{R}_{+}^{N}\right)}$ is a norm on $\stackrel{\circ}{W}_{\alpha, \beta}^{m, p}\left(\mathbb{R}_{+}^{N}\right)$ which is equivalent to the full norm $\|\cdot\|_{W_{\alpha, \beta}^{m, p}\left(\mathbb{R}_{+}^{N}\right)}$.

1.3. The spaces of traces. In order to define the traces of functions of $W_{\alpha, \beta}^{m, p}\left(\mathbb{R}_{+}^{N}\right)$, we introduce for any $\sigma \in(0,1)$ the space

$$
\begin{aligned}
W_{0}^{\sigma, p}\left(\mathbb{R}^{N}\right)=\{u \in & \mathcal{D}^{\prime}\left(\mathbb{R}^{N}\right) \mid w^{-\sigma} u \in L^{p}\left(\mathbb{R}^{N}\right), \\
& \left.\sum_{i=1}^{N} \int_{0}^{\infty} t^{-1-\sigma p} d t \int_{\mathbb{R}^{N}}\left|u\left(x+t e_{i}\right)-u(x)\right|^{p} d x<\infty\right\},
\end{aligned}
$$

where

$$
w= \begin{cases}\varrho & \text { if } N / p \neq \sigma \\ \varrho(\lg \varrho)^{1 / \sigma} & \text { if } N / p=\sigma\end{cases}
$$

and $e_{1}, \ldots, e_{N}$ is the canonical basis of $\mathbb{R}^{N}$. It is a reflexive Banach space equipped with its natural norm

$\|u\|_{W_{0}^{\sigma, p}\left(\mathbb{R}^{N}\right)}=\left(\left\|\frac{u}{w^{\sigma}}\right\|_{L^{p}\left(\mathbb{R}^{N}\right)}^{p}+\sum_{i=1}^{N} \int_{0}^{\infty} t^{-1-\sigma p} d t \int_{\mathbb{R}^{N}}\left|u\left(x+t e_{i}\right)-u(x)\right|^{p} d x\right)^{1 / p}$,

which is equivalent to the norm

$$
\left(\left\|\frac{u}{w^{\sigma}}\right\|_{L^{p}\left(\mathbb{R}^{N}\right)}^{p}+\int_{\mathbb{R}^{N} \times \mathbb{R}^{N}} \frac{|u(x)-u(y)|^{p}}{|x-y|^{N+\sigma p}} d x d y\right)^{1 / p} .
$$

If $u$ is a function defined on $\mathbb{R}_{+}^{N}$, we denote its traces on $\Gamma=\mathbb{R}^{N-1}$ by

$$
\gamma_{0} u\left(x^{\prime}\right)=u\left(x^{\prime}, 0\right), \ldots, \gamma_{j} u\left(x^{\prime}\right)=\frac{\partial^{j} u}{\partial x_{N}^{j}}\left(x^{\prime}, 0\right), \quad x^{\prime} \in \mathbb{R}^{N-1} .
$$


In the same way as in [5], we can prove the following trace lemma:

Lemma 1.1. For any integer $m \geq 1$ and real number $\alpha$, the mapping

$$
\gamma: \mathcal{D}\left(\overline{\mathbb{R}_{+}^{N}}\right) \rightarrow \prod_{j=0}^{m-1} \mathcal{D}\left(\mathbb{R}^{N-1}\right), \quad u \mapsto\left(\gamma_{0} u, \ldots, \gamma_{m-1} u\right)
$$

can be extended to a continuous linear mapping, still denoted by $\gamma$, from $W_{\alpha}^{m, p}\left(\mathbb{R}_{+}^{N}\right)$ onto $\prod_{j=0}^{m-1} W_{\alpha}^{m-j-1 / p, p}\left(\mathbb{R}^{N-1}\right)$. Moreover

$$
\operatorname{Ker} \gamma=\stackrel{\circ}{W}_{\alpha}^{m, p}\left(\mathbb{R}_{+}^{N}\right) \text {. }
$$

Proof. See [2].

1.4. The mapping $T_{t}$. We consider the general case of constructing the transformation $T_{t}$. Let $D$ be a domain in $\mathbb{R}^{N}$ with the boundary $\partial D$ piecewise $C^{k}$ for a given integer $k \geq 0$. Let $T_{t}$ be a one-to-one mapping from $\bar{D}$ onto $\bar{D}$ such that

$$
T_{t} \text { and } T_{t}^{-1} \text { belong to } C^{k}\left(\bar{D} ; \mathbb{R}^{N}\right)
$$

and

$$
t \mapsto T_{t}(x), T_{t}^{-1}(x) \in C([0, \varepsilon))^{N}, \quad \forall x \in \bar{D},
$$

thus the function $(t, x) \mapsto T_{t}(x)$ belongs to the space $C\left([0, \varepsilon) ; C^{k}\left(\bar{D} ; \mathbb{R}^{N}\right)\right)$ denoted by $C\left(0, \varepsilon ; C^{k}\left(\bar{D} ; \mathbb{R}^{N}\right)\right)$. For any $X \in \bar{D}$ and $t>0$ the point $x(t)=$ $T_{t}(X)$ moves along the trajectory $x(\cdot)$ with the velocity

$$
\left\|\frac{d}{d t} x(t)\right\|_{\mathbb{R}^{N}}=\left\|\frac{\partial}{\partial t} T_{t}(X)\right\|_{\mathbb{R}^{N}}
$$

and satisfies the system of ordinary differential equations

$$
\frac{d}{d t} x(t)=V(t, x(t))
$$

It is obvious that the speed vector field $V(t, x)$ takes the form

$$
V(t, x)=\left(\frac{\partial}{\partial t} T_{t}\right) \circ T_{t}^{-1}(x)
$$

From (1.13) and (1.14) the vector field $V(t)$, defined as $V(t)(x)=V(t, x)$, satisfies the relation

$$
V \in C\left(0, \varepsilon ; C^{k}\left(\bar{D} ; \mathbb{R}^{N}\right)\right) .
$$

If $V$ is a vector field such that (1.18) holds, then the transformation $T_{t}$ depending on $V$ and such that conditions (1.13) and (1.14) are satisfied, is defined by (1.2).

THEOREM 1.4. Let $D$ be a bounded domain in $\mathbb{R}^{N}$ with piecewise smooth boundary $\partial D$, and $V \in C\left(0, \varepsilon ; C^{k}\left(\bar{D}, \mathbb{R}^{N}\right)\right)$ be a given vector field which 
satisfies

$$
V(t, x) \cdot n(x)=0 \quad \text { for a.e. } x \in \partial D,
$$

and

$$
\begin{aligned}
& \text { if } n=n(x) \text { is not defined at a singular point } x \in \partial D, \text { then we set } \\
& V(t, x)=0 .
\end{aligned}
$$

Then there exists an open interval $I, 0 \in I$, and a one-to-one transformation $T_{t}(V): \mathbb{R}^{N} \rightarrow \mathbb{R}^{N}$ such that $T_{t}(V)$ maps $\bar{D}$ onto $\bar{D}$. Furthermore $T_{t}(V)$ satisfies conditions (1.13), (1.14), (1.2). In particular the vector field $V$ can be written in the form

$$
V=\partial_{t} T_{t}(V) \circ T_{t}(V)^{-1}
$$

On the other hand, if $T_{t}$ is a transformation of $\bar{D}, T_{t}$ satisfies (1.13), (1.14) and $V$ is defined by the formula

$$
V=\partial_{t} T_{t} \circ T_{t}^{-1}
$$

then $V \in C\left(0, \varepsilon ; C^{k}\left(\bar{D}, \mathbb{R}^{N}\right)\right)$ and (1.19) holds for the field $V$. Given a vector field $V$ with the above properties, the transformation $T_{t}(X)=x(t, X)$ can be defined, in an equivalent way, as a local solution to the system of ordinary differential equations (1.2), that is, $T_{t}=T_{t}(V)$.

Now, we are interested in the case of a domain $D$ which is not bounded. $\mathcal{D}^{k}\left(\mathbb{R}^{N} ; \mathbb{R}^{N}\right)$ denotes the space of compactly supported vector fields with the uniform convergence in $C^{k}$; we refer the reader to [8] for the details.

Definition 1.1. Let $D$ be a domain in $\mathbb{R}^{N}$ whose boundary $\partial D$ is piecewise $C^{k}, k \geq 1$. It is supposed that the outward unit normal field $n$ exists a.e. on $\partial D$, i.e. except for singular points $\bar{x}$ of $\partial D$. The following notation is used:

$$
\begin{array}{r}
V^{k}(D)=\left\{V \in \mathcal{D}^{k}\left(\mathbb{R}^{N} ; \mathbb{R}^{N}\right) \mid\langle V, n\rangle_{\mathbb{R}^{N}}=0 \text { on } \partial D\right. \text { except at singular points } \\
\bar{x} \text { of } \partial D, V(\bar{x})=0 \text { for all singular points } \bar{x}\} .
\end{array}
$$

$V^{k}(D)$ is equipped with the topology induced by $\mathcal{D}^{k}\left(\mathbb{R}^{N} ; \mathbb{R}^{N}\right)$.

So, if $V \in C\left(0, \varepsilon ; V^{k}(D)\right)$, then there exists a compact set $\bar{O}$ in $\mathbb{R}^{N}$ such that the support of $V(t)$ is included in $\bar{O}$ for all $0 \leq t \leq \varepsilon$. So, we have the following theorem:

TheOREM 1.5. Let $D$ be a bounded domain in $\mathbb{R}^{N}$ with piecewise smooth boundary $\partial D$, and $V \in C\left(0, \varepsilon ; V^{k}(D)\right)$ be a vector field. Then there exists an interval $I=[0, \delta), 0<\delta<\varepsilon$, and a one-to-one transformation $T_{t}(V)$ for each $t \in I$ which maps $\bar{D}$ onto $\bar{D}$ and has all the properties of Theorem 1.4. 
1.5. Sobolev spaces and boundary value problems $(\mathrm{TP})$ and $(\mathrm{PP})$ in perturbed domains. We already introduced the Sobolev weighted spaces in a fixed domain. Now, we are interested in the definition of Sobolev spaces with corresponding weights in a perturbed domain. The most important property is the existence of traces and the trace theorem.

Definition 1.2. We write $u_{t} \in W_{0}^{1,2}\left(\Omega_{t}\right)$ iff $u^{t}=u_{t} \circ T_{t} \in W_{0}^{1,2}(\Omega)$ and the corresponding norm is defined by

$$
\int_{\Omega}\left\|\nabla u^{t}\right\|^{2} d x=\int_{\Omega_{t}}\left\|D T_{t} \circ T_{t}^{-1} \cdot \nabla u_{t}\right\|^{2}|\gamma(t)|^{-1} d y .
$$

The trace on $\Gamma_{t}$ is defined in the following way: $u_{t}=0$ on $\Gamma_{t}$ iff $u^{t}=0$ on $\Gamma$. We set $\left.\stackrel{\circ}{W}_{0}^{1,2}\left(\Omega_{t}\right)=\overline{\mathcal{D}\left(\Omega_{t}\right.}\right)^{\|\cdot\|_{W_{0}^{1,2}(\Omega)}}=\left\{u_{t} \in W_{0}^{1,2}\left(\Omega_{t}\right) \mid u_{t}=0\right.$ on $\left.\Gamma_{t}\right\}$ with the corresponding norm

$$
\begin{aligned}
\left\|u_{t}\right\|_{W_{0}^{1,2}\left(\Omega_{t}\right)}= & \left\{\int_{\Omega_{t}}\left\|D T_{t} \circ T_{t}^{-1} \cdot \nabla u_{t}\right\|^{2}|\gamma(t)|^{-1} d y\right. \\
& \left.+\int_{\Omega_{t}}\left\|u_{t} \circ T_{t}^{-1}\right\|^{2}\left(\varrho \circ T_{t}^{-1}\right)^{-2}|\gamma(t)|^{-1} d y\right\}^{1 / 2} .
\end{aligned}
$$

Definition 1.3. We write $g_{t} \in W_{0}^{1 / 2,2}\left(\Gamma_{t}\right)$ iff $g_{t} \circ T_{t}=g^{t} \in W_{0}^{1 / 2,2}(\Gamma)$ with the norm defined by

$$
\int_{\Gamma_{t} \times \Gamma_{t}} \frac{1}{w^{4}(t)} \frac{\mid g_{t}\left(x_{t}^{\prime}\right)-g_{t}\left(\left.y^{\prime}(t)\right|^{2}\right.}{\left|S_{t} x_{t}^{\prime}-S_{t} y_{t}^{\prime}\right|^{2}} d x_{t}^{\prime} d y_{t}^{\prime}=\int_{\Gamma \times \Gamma} \frac{\left|g^{t}\left(x^{\prime}\right)-g^{t}\left(y^{\prime}\right)\right|^{2}}{\left|x^{\prime}-y^{\prime}\right|^{2}} d x^{\prime} d y^{\prime}<\infty,
$$

where $w(t)=\left|\operatorname{det}\left(D T_{t}\right)\right|\left\|\left(D T^{-1}\right)^{*} \cdot n\right\|_{\mathbb{R}^{N}}, x_{t}^{\prime}=T_{t} x^{\prime}, y_{t}^{\prime}=T_{t} y^{\prime}, M\left(T_{t}\right)=$ $\operatorname{det}\left(D T_{t}\right)^{*} D T^{-1}$ is the cofactor matrix of the Jacobian $D T_{t}$, and $S_{t}=T_{t}^{-1}$.

First we are interested in weak differentiability with respect to $t$.

Proposition 1.1. Let $f \in \stackrel{\circ}{W}_{1}^{0,2}(D) \subset W_{0}^{-1,2}(D)$. Let $V \in C(0, \varepsilon$, $\left.\mathcal{D}^{k}\left(\mathbb{R}_{+}^{N} ; \mathbb{R}^{N}\right)\right)$ be given, $k \geq 1$. Then the mapping $t \mapsto f \circ T_{t}$ is weakly differentiable in the space $W_{0}^{-1,2}(D)$.

Proof. Let $\varphi \in \stackrel{\circ}{W}_{0}^{1,2}\left(\mathbb{R}_{+}^{N}\right) \subset W_{-1}^{0,2}\left(\mathbb{R}_{+}^{N}\right)$ be given and define $\lambda(t)=$ $\gamma(t)^{-1} \circ T_{t}^{-1}=\gamma(t)^{-1} \circ S_{t}$. We have

$$
\frac{1}{t} \int_{D}\left(f \circ T_{t}-f\right) \varphi d x=\frac{1}{t} \int_{D} f\left(\lambda(t) \varphi \circ S_{t}-\varphi\right) d x .
$$

Furthermore

$$
\left.\frac{1}{t}\left(\lambda(t) \varphi \circ S_{t}-\varphi\right)=\lambda(t) \frac{1}{t}\left(\varphi \circ S_{t}-\varphi\right)+\frac{1}{t}(\lambda(t)-1) \varphi\right),
$$

and the right hand side converges to $-\nabla \varphi \cdot V(0)+\lambda^{\prime}(0) \varphi$ strongly in $W_{-1}^{0,2}\left(\mathbb{R}_{+}^{N}\right)$ as $t \rightarrow 0$. Moreover, it is evident that $\lambda^{\prime}(0)=-\operatorname{div} V(0)$. 
Since $S_{t}$ is associated with the speed vector field $-V_{t}$, we have $\int_{D} \frac{1}{t}\left(f \circ T_{t}-f\right) \varphi d x \rightarrow-\int_{D} f \operatorname{div}(\varphi V(0)) d x=\langle f \cdot V(0), \varphi\rangle_{W_{0}^{-1,2}(D) \times \stackrel{\circ}{W}_{0}^{1,2}(D)}$ as $t \rightarrow 0$; this proves the proposition.

We are interested in the differentiability of the mapping $t \mapsto f \circ T_{t}$, where $f$ is a measure or a distribution which cannot be represented by any integrable function.

Definition 1.4. Let $h_{t} \in W_{0}^{-1,2}\left(\Omega_{t}\right)$ and $\varphi \in \stackrel{\circ}{W}_{0}^{1,2}(\Omega)$. Then we define

$$
\left\langle\tau h_{t}, \varphi\right\rangle_{W_{0}^{-1,2}(\Omega) \times \stackrel{\circ}{W}_{0}^{1,2}(\Omega)}=\left\langle h_{t}, \gamma^{-1}(t) \varphi \circ T_{t}^{-1}\right\rangle_{W_{0}^{-1,2}\left(\Omega_{t}\right) \times \stackrel{\circ}{W}_{0}^{1,2}\left(\Omega_{t}\right)} .
$$

REMARK 1.1. If $h_{t} \in L^{2}\left(\Omega_{t}\right)$, then $\varphi \circ T_{t}^{-1} \in \stackrel{\circ}{W}_{0}^{1,2}\left(\Omega_{t}\right)$ for all $\varphi \in$ $\stackrel{\circ}{W}_{0}^{1,2}(\Omega)$, and we have

$$
\begin{aligned}
\left\langle h_{t}, \gamma(t)^{-1} \varphi \circ T_{t}^{-1}\right\rangle_{W_{0}^{-1,2}}\left(\Omega_{t}\right) \times \stackrel{\circ}{W}_{0}^{1,2}\left(\Omega_{t}\right) & =\int_{\Omega_{t}} h_{t}(x) \cdot \gamma(t)^{-1} \varphi \circ T_{t}^{-1}(x) d x \\
& =\int_{\Omega} h_{t} \circ T_{t}(X) \varphi(X) d X=\left\langle h_{t} \circ T_{t}, \varphi\right\rangle_{W_{0}^{-1,2}(\Omega) \times \stackrel{\circ}{W}_{0}^{1,2}(\Omega)},
\end{aligned}
$$

where $h^{t}=h_{t} \circ T_{t}$ and $h_{t} \in L^{2}\left(\Omega_{t}\right)$.

Proposition 1.2. (i) If $h_{t} \in W_{0}^{-1,2}\left(\Omega_{t}\right)$, then

$$
h_{t}=\operatorname{div} \mathbf{F}, \quad \mathbf{F}=\left(f_{1}, \ldots, f_{N}\right),
$$

with $f_{i} \in L^{2}\left(\Omega_{t}\right), i=0, \ldots, n$.

(ii) $h^{t}=\gamma(t)^{-1} \operatorname{div}\left(D T_{t}^{-1} \mathbf{F} \circ T_{t}\right)$.

(iii) In particular if $h \in W_{0}^{-1,2}(D)$, where $\Omega \subset D, \Omega_{t} \subset D$, then $h^{t} \in$ $W_{0}^{-1,2}(D)$ and

$$
\frac{h-h_{t}}{t} \rightarrow \dot{h} \quad \text { weakly in } W_{0}^{-1,2}(D) .
$$

Proof. This follows from the above.

By an application of the transport technique to our problem (1.1) defined in $\Omega_{t}$, we get

$$
\left\langle\Delta u_{t}, \psi\right\rangle_{W_{0}^{-1,2}\left(\Omega_{t}\right) \times \stackrel{\circ}{W}_{0}^{1,2}\left(\Omega_{t}\right)}=\left\langle\operatorname{div}\left(A(t) \nabla u^{t}\right), \psi \circ T_{t}\right\rangle_{W_{0}^{-1,2}(\Omega) \times \stackrel{\circ}{W}_{0}^{1,2}(\Omega)} .
$$

Let $\varphi=\psi \circ T_{t} \in \stackrel{\circ}{W}_{0}^{1,2}(\Omega)$. Then

$$
\left\langle\Delta u_{t}, \varphi \circ T_{t}^{-1}\right\rangle_{W_{0}^{-1,2}\left(\Omega_{t}\right) \times \stackrel{\circ}{W}_{0}^{1,2}\left(\Omega_{t}\right)}=\left\langle\operatorname{div}\left(A(t) \nabla u^{t}\right), \varphi\right\rangle_{W_{0}^{-1,2}(\Omega) \times \stackrel{\circ}{W}_{0}^{1,2}(\Omega)}
$$

provided that

$$
-\Delta u_{t}=h_{t} \quad \text { in } \Omega_{t},
$$

whence $-\operatorname{div}\left(A(t) \nabla u^{t}\right)=\gamma(t) h^{t}$ in $\Omega$. 
For problem (PP), $-\Delta u_{t}=f_{t}$ in $\Omega_{t}$ thus

$$
-\operatorname{div}\left(A(t) \nabla u^{t}\right)=\gamma(t) f^{t} \quad \text { in } \Omega .
$$

Therefore, problem (PP)

$$
\begin{array}{ll}
-\Delta u_{t}=f_{t} & \text { in } \Omega_{t}, \\
u_{t}=g_{t} & \text { on } \Gamma_{t},
\end{array}
$$

is transported to the fixed domain $\Omega$ and problem (TP) becomes

$$
\begin{array}{ll}
-\operatorname{div}\left(A(t) \nabla u^{t}\right)=\gamma(t) f^{t} & \text { in } \Omega, \\
u^{t}=g^{t} & \text { on } \Gamma,
\end{array}
$$

where $g^{t}=g_{t} \circ T_{t}$.

2. Problem (TP). First, we investigate the existence of transported solutions in the fixed domain $\Omega$ satisfying the equations

$$
\begin{array}{ll}
-\operatorname{div}\left(A(t) \nabla u^{t}\right)=F^{t} & \text { in } \Omega, \\
u^{t}=g^{t} & \text { on } \partial \Omega .
\end{array}
$$

We define a bilinear form by

$$
B(u, v)=\int_{\Omega} A(t) \nabla u \cdot \nabla v .
$$

Since $\gamma(0)=1$, for sufficiently small $\delta>0$ we have $\gamma(t)>1 / 2$ for all $t \in(-\delta, \delta)$ and the bilinear form $B$ is uniformly elliptic, i.e.,

$$
B(u, u) \geq c\|\nabla u\|_{2}^{2}
$$

for some positive constant $c>0$.

TheOREM 2.1. Let $\gamma(t) f^{t} \in W_{0}^{-1,2}\left(\mathbb{R}_{+}^{N}\right)$ and $g^{t} \in W_{0}^{1 / 2,2}\left(\mathbb{R}^{N-1}\right)$. Then problem (TP) admits one and only one solution $u^{t} \in W_{0}^{1,2}\left(\mathbb{R}_{+}^{N}\right)$, and there exists a constant $c>0$, independent of $t \in(-\delta, \delta), f^{t}$ and $g^{t}$, such that

$$
\left\|u^{t}\right\|_{W_{0}^{1,2\left(\mathbb{R}_{+}^{N}\right)}} \leq c\left(\left\|\gamma(t) f^{t}\right\|_{W_{0}^{-1,2}\left(\mathbb{R}_{+}^{N}\right)}+\left\|g^{t}\right\|_{W_{0}^{1 / 2,2}\left(\mathbb{R}_{+}^{N-1}\right)}\right) .
$$

Moreover, if $\gamma(t) f^{t} \in W_{m}^{m-1,2}\left(\mathbb{R}_{+}^{N}\right)$ and $g^{t} \in W_{m}^{m+1 / 2,2}\left(\mathbb{R}^{N-1}\right)$ for some integer $m \geq 1$, then $u \in W_{m}^{m+1,2}\left(\mathbb{R}_{+}^{N}\right)$, and there exists a constant $c=c(m)$ such that

$$
\left\|u^{t}\right\|_{W_{m}^{m+1,2}\left(\mathbb{R}_{+}^{N}\right)} \leq c\left(\left\|\gamma(t) f^{t}\right\|_{W_{m}^{m-1,2}\left(\mathbb{R}_{+}^{N}\right)}+\left\|g^{t}\right\|_{W_{m}^{m+1 / 2,2}\left(\mathbb{R}_{+}^{N-1}\right)}\right) .
$$

Since the non-homogeneous Dirichlet boundary condition $g^{t}$ is given by the composition $g_{t} \circ T_{t}$, we can always consider the equivalent problem with the homogeneous Dirichlet condition. First, we define $G^{t}$ as an extension such that $g^{t}=G^{t}$ on $\Gamma^{t}$ and $G^{t} \in W_{0}^{1,2}\left(\mathbb{R}_{+}^{N}\right)$. Actually, we have the standard result. 
Proposition 2.1. The solution $u^{t} \in W_{0}^{1,2}\left(\mathbb{R}_{+}^{N}\right)$ of problem (TP) takes the form $u^{t}=w^{t}+G^{t}$, where $w^{t} \in \stackrel{\circ}{W}_{0}^{1,2}\left(\mathbb{R}_{+}^{N}\right)$ is a unique solution to the following problem:

$$
B\left(w^{t}, \varphi\right)=\left\langle f^{t}-\Delta G^{t}, \varphi\right\rangle, \quad \forall \varphi \in \stackrel{\circ}{W}_{0}^{1,2}\left(\mathbb{R}_{+}^{N}\right) .
$$

From the Lax-Milgram lemma it follows that the variational problem (2.4) admits a unique solution $w^{t} \in \stackrel{\circ}{W}_{0}^{1,2}\left(\mathbb{R}_{+}^{N}\right)$. The bilinear and linear forms in (2.4) are continuous and the bilinear form is $W_{0}^{1,2}\left(\mathbb{R}_{+}^{N}\right)$-elliptic. Applying the Schwartz reflection principle and induction we get the required regularity.

3. Problem (PP). Now, we deal with the perturbed problem

$$
\begin{array}{ll}
-\Delta u_{t}=f_{t} & \text { in } \Omega_{t}=T_{t}\left(\mathbb{R}_{+}^{N}\right), \\
u_{t}=g_{t} & \text { on } \Gamma_{t}=T_{t}\left(\mathbb{R}^{N-1}\right) .
\end{array}
$$

Proposition 3.1. If $f \in \stackrel{\circ}{W}_{1}^{0,2}\left(\mathbb{R}_{+}^{N}\right)$, then

$$
\frac{F^{t}-f}{t} \rightarrow \operatorname{div} V f+\dot{f} \quad \text { weakly in } W_{0}^{-1,2}\left(\mathbb{R}_{+}^{N}\right) \text {. }
$$

Proof. We have

$$
\begin{aligned}
\frac{1}{t}\left\langle F^{t}-\right. & f, \varphi\rangle-\langle\dot{f}-\operatorname{div} V f, \varphi\rangle \\
& =\frac{1}{t}\left\langle-\operatorname{div}\left(A(t) \nabla u^{t}\right)+\operatorname{div} \nabla u, \varphi\right\rangle+\langle\operatorname{div} V f, \varphi\rangle \\
& =\frac{1}{t}\left\langle(A(t)-I) \nabla u^{t}, \nabla \varphi\right\rangle-\frac{1}{t}\left\langle\nabla\left(u^{t}-u\right), \nabla \varphi\right\rangle+\langle\operatorname{div} V f, \varphi\rangle \\
& \rightarrow\left\langle A^{\prime}(0) \nabla u, \nabla \varphi\right\rangle-\langle\nabla \dot{u}, \nabla \varphi\rangle+\langle\operatorname{div} V f, \varphi\rangle=\langle\dot{f}, \varphi\rangle+\langle\operatorname{div} V f, \varphi\rangle .
\end{aligned}
$$

For $f \in W_{1}^{0,2}\left(\mathbb{R}_{+}^{N}\right)$ it follows that $\dot{f} \in W_{0}^{-1,2}\left(\mathbb{R}_{+}^{N}\right)$ and also

$$
\frac{f^{t}-f}{t}-\dot{f} \rightarrow 0 \quad \text { weakly in } W_{0}^{-1,2}\left(\mathbb{R}_{+}^{N}\right) \text {. }
$$

For $g \in W_{1}^{3 / 2,2}(\Gamma)$ with $\dot{g} \in W_{0}^{1 / 2,2}(\Gamma)$ it follows that

$$
\frac{g^{t}-g}{t}-\dot{g} \rightarrow 0 \quad \text { weakly in } W_{0}^{1 / 2,2}(\Gamma) \text {. }
$$

ThEOREM 3.1. (i) Let $f_{t} \in W_{0}^{-1,2}\left(\Omega_{t}\right)$ and $g_{t} \in W_{0}^{1 / 2,2}\left(\Gamma_{t}\right)$. Then problem (PP) admits a unique solution $u^{t} \in W_{0}^{1}\left(\Omega_{t}\right)$, and there exists a constant $c$, not depending on $f_{t}$ and $g_{t}$, such that

$$
\left\|u_{t}\right\|_{W_{0}^{1,2}\left(\Omega_{t}\right)} \leq c\left(\left\|f_{t}\right\|_{W_{0}^{-1,2}\left(\Omega_{t}\right)}+\left\|g_{t}\right\|_{W_{0}^{1 / 2,2}\left(\Gamma_{t}\right)}\right) .
$$

(ii) Moreover, if $f_{t} \in W_{1}^{1,2}(\Omega)$ and $g_{t} \in W_{1}^{3 / 2,2}(\Gamma)$ then problem $(\mathrm{PP})$ admits a unique solution $u_{t} \in W_{1}^{2}(\Omega)$, and there exists a constant $c$, 
not depending on $f_{t}$ and $g_{t}$, such that

$$
\left\|u_{t}\right\|_{W_{1}^{2}\left(\Omega_{t}\right)} \leq c\left(\left\|f_{t}\right\|_{W_{1}^{0}\left(\Omega_{t}\right)}+\left\|g_{t}\right\|_{W_{1}^{3 / 2}\left(\Gamma_{t}\right)}\right) .
$$

Proof. First, we use the variational formulation

$$
\begin{aligned}
\left\langle f_{t}, \psi\right\rangle_{W_{0}^{-1,2}\left(\Omega_{t}\right) \times \stackrel{\circ}{W}_{0}^{1,2}\left(\Omega_{t}\right)}=\left\langle\gamma(t) f_{t}, \gamma^{-1} \varphi \circ T_{t}^{-1}\right\rangle_{W_{0}^{-1,2}\left(\Omega_{t}\right) \times \stackrel{\circ}{W}_{0}^{1,2}\left(\Omega_{t}\right)} \\
=\left\langle\gamma(t) \Delta u_{t}, \gamma^{-1}(t) \varphi \circ T_{t}^{-1}\right\rangle_{W_{0}^{-1,2}\left(\Omega_{t}\right) \times \stackrel{\circ}{W}_{0}^{1,2}\left(\Omega_{t}\right)} \\
=\left\langle\operatorname{div}\left(A(t) \nabla u^{t}\right), \varphi\right\rangle_{W_{0}^{-1,2}(\Omega) \times \stackrel{\circ}{W}_{0}^{1,2}(\Omega)}=\left\langle f_{t}, \varphi\right\rangle_{W_{0}^{-1,2}(\Omega) \times \stackrel{\circ}{W}_{0}^{1,2}(\Omega)},
\end{aligned}
$$

and Theorem 3.1 is a consequence of Theorem 2.1. As before, we apply the Lax-Milgram lemma. It is sufficient to consider the transported problem. We set $z^{t}=u^{t}-G^{t}$. Then we look for the solution $z^{t}$ of the Laplace equation with the homogeneous Dirichlet condition. Therefore, we have the homogeneous Dirichlet problem for $z_{t} \in \stackrel{\circ}{W}_{0}^{1}\left(\Omega_{t}\right)$ and the estimate (3.1) follows.

4. Material derivative. We want to prove the existence of the strong limit $u^{t} \rightarrow u \in W_{0}^{1,2}$ and the weak limit for

$$
\frac{u^{t}-u}{t} \rightarrow \dot{u} \in W_{0}^{1,2}\left(\mathbb{R}_{+}^{N}\right) .
$$

Defining

$$
w^{t}=\frac{u^{t}-u}{t}-\dot{u}
$$

we obtain the following equation:

$$
\begin{array}{ll}
-\Delta w^{t}=\operatorname{div}\left[\frac{A(t)-I}{t} \nabla u^{t}-A^{\prime}(0) \nabla u\right]+\frac{f^{t}-f}{t}-\dot{f} & \text { in } \Omega, \\
w^{t}=\frac{g^{t}-g}{t}-\dot{g} & \text { on } \Gamma .
\end{array}
$$

The weak formulation of (4.2) is the following:

$$
\begin{aligned}
\int_{\mathbb{R}_{+}^{N}} \nabla w_{t} \cdot \nabla \phi= & \int_{\mathbb{R}_{+}^{N}}\left[\frac{A(t)-I}{t} \nabla u^{t} \cdot \nabla \phi-A^{\prime}(0) \nabla u \nabla \phi\right] \\
& +\int_{\mathbb{R}_{+}^{N}}\left[\frac{f^{t}-f}{t}-\dot{f}\right] \phi, \quad \forall \phi \in \stackrel{\circ}{W}_{0}^{1,2}\left(\mathbb{R}_{+}^{N}\right) .
\end{aligned}
$$

The goal of this section is to prove the following convergences:

$$
\begin{array}{ll}
w^{t}=\frac{u^{t}-u}{t}-\dot{u} \rightarrow 0 & \text { as } t \rightarrow 0, \text { weakly in } W_{0}^{1,2}\left(\mathbb{R}_{+}^{N}\right), \\
w_{t}=\frac{g^{t}-g}{t}-\dot{g} \rightarrow 0 & \text { as } t \rightarrow 0, \text { weakly in } W_{0}^{1 / 2,2}\left(\mathbb{R}^{N-1}\right) .
\end{array}
$$


It is sufficient to prove that

$$
\frac{A(t)-I}{t} \nabla u^{t}-A^{\prime}(0) \nabla u \rightarrow 0 \quad \text { as } t \rightarrow 0, \text { weakly in } L^{2}\left(\mathbb{R}_{+}^{N}\right)^{N},
$$

since for the right hand side we have by our assumptions

$$
\frac{f^{t}-f}{t}-\dot{f} \rightarrow 0 \quad \text { as } t \rightarrow 0 \text {, weakly in } W_{0}^{-1,2}\left(\mathbb{R}_{+}^{N}\right),
$$

where $A^{\prime}(0)=-D V-(D V)^{*}+\operatorname{div} V I$.

Let $\varphi=u^{t}-u$ be a test function in the variational formulation. Then

$$
\int_{\mathbb{R}_{+}^{N}} A(t)\left|\nabla\left(u^{t}-u\right)\right|^{2}-(A(t)-I) \nabla u \cdot \nabla\left(u^{t}-u\right)=\left\langle f^{t}-f, u^{t}-u\right\rangle .
$$

Since the field $V$ is compactly supported in $\mathbb{R}^{N}$, it follows that

$$
\begin{aligned}
& \int_{\mathbb{R}_{+}^{N}} A(t)\left|\nabla\left(u^{t}-u\right)\right|^{2} \\
& \quad \leq \int_{\mathbb{R}_{+}^{N}}\left|(A(t)-I) \nabla u \cdot \nabla\left(u^{t}-u\right)\right|+\left\|f^{t}-f\right\|_{W_{0}^{-1,2}}\left\|u^{t}-u\right\|_{W_{0}^{1,2}} \\
& \quad \leq c(t)\|\nabla u\|_{L^{2}\left(\mathbb{R}_{+}^{N}\right)}\left\|\nabla\left(u^{t}-u\right)\right\|_{L^{2}}+\left\|f^{t}-f\right\|_{W_{0}^{-1,2}}\left\|u^{t}-u\right\|_{W_{0}^{1,2}} .
\end{aligned}
$$

From the properties of $A(t)=I+O(t)$, for $|t|$ small enough we have

$$
\frac{1}{2}\left\|u^{t}-u\right\|_{W_{0}^{1,2}} \leq c(t)\|\nabla u\|_{L^{2}}+c\left\|f^{t}-f\right\|_{W_{0}^{-1,2}}
$$

Since $f \in W_{0}^{-1,2}$ and we have shown that $f^{t}$ is strongly continuous with respect to $t$, i.e. $f^{t} \rightarrow f$ in $W_{0}^{-1,2}, g^{t} \rightarrow g$ in $W_{0}^{1 / 2,2}$, it follows that $u^{t} \rightarrow$ $u$ in $W_{0}^{1,2}\left(\mathbb{R}_{+}^{N}\right)$ provided $c(t) \rightarrow 0$.

Since $V$ is compactly supported, i.e. supp $V \subset B(R)$ for some $R$, the first term on the right hand side of (4.7) takes the form

$$
\begin{aligned}
\int_{\Omega}(A(t)-I) \nabla u \cdot \nabla \varphi & =\int_{B(R)}(A(t)-I) \nabla u \cdot\left(\nabla u^{t}-\nabla u\right) \\
& \leq c(t)\|\nabla u\|_{L^{2}}\left\|\nabla\left(u^{t}-u\right)\right\|_{W_{0}^{-1,2}}
\end{aligned}
$$

and it follows, in view of the properties of the mapping $T_{t}(V)$, that $c(t) \rightarrow 0$, which implies that (4.5) and (4.6) hold and $u^{t} \rightarrow u$ in $W_{0}^{1,2}\left(\mathbb{R}_{+}^{N}\right)$.

Therefore, we have the following result.

Corollary 4.1. If $f \in W_{0}^{-1,2}\left(\mathbb{R}_{+}^{N}\right)$ and $g \in W_{0}^{1 / 2,2}\left(\mathbb{R}^{N-1}\right)$ then the material derivative $\dot{u} \in W_{0}^{1,2}\left(\mathbb{R}_{+}^{N}\right)$ is given by a unique solution to problem (1.3). 
5. Existence of material and shape derivatives for the Dirichlet Laplacian in a half-space. We show the existence and uniqueness of solutions to the following two problems:

$$
\begin{aligned}
& -\Delta \dot{u}=\operatorname{div} V f+\dot{f}+\operatorname{div}\left(A^{\prime}(0) \nabla u\right), \\
& \dot{u}=\dot{g},
\end{aligned}
$$

and

$$
\begin{aligned}
& -\Delta u^{\prime}=f^{\prime}, \\
& u^{\prime}=g^{\prime} .
\end{aligned}
$$

As a consequence of the general theory for Dirichlet problems we have the following theorems:

Theorem 5.1. Let $f \in W_{1}^{0,2}\left(\mathbb{R}_{+}^{N}\right)$ and $g \in W_{1}^{3 / 2,2}\left(\mathbb{R}^{N-1}\right)$. Then problem (5.1) admits a unique solution $\dot{u} \in W_{0}^{1,2}\left(\mathbb{R}_{+}^{N}\right)$, and there exists a constant $c>0$, independent of $t \in(-\delta, \delta)$, such that

$$
\|\dot{u}\|_{W_{0}^{1,2}\left(\mathbb{R}_{+}^{N}\right)} \leq c\left(\|f\|_{W_{1}^{0,2}\left(\mathbb{R}_{+}^{N}\right)}+\|g\|_{W_{1}^{3 / 2,2}\left(\mathbb{R}^{N-1}\right)}\right) .
$$

TheOREM 5.2. Let $f \in W_{1}^{0,2}\left(\mathbb{R}_{+}^{N}\right)$ and $g \in W_{1}^{3 / 2,2}\left(\mathbb{R}^{N-1}\right)$. Then problem (5.2) admits a unique solution $u^{\prime} \in W_{0}^{1}\left(\mathbb{R}_{+}^{N}\right)$, and there exists a constant $c>0$, independent of $t \in(-\delta, \delta)$, such that

$$
\left\|u^{\prime}\right\|_{W_{1}^{0,2}\left(\mathbb{R}_{+}^{N}\right)} \leq c\left(\|f\|_{W_{1}^{0,2}\left(\mathbb{R}_{+}^{N}\right)}+\|g\|_{W_{1}^{3 / 2,2}\left(\mathbb{R}^{N-1}\right)}\right) .
$$

6. Shape derivatives of functionals. We can use the above results to establish the shape differentiability of shape functionals. We start with the energy functional

$$
\mathcal{E}(\Omega)=\frac{1}{2} \int_{\Omega}\left|\nabla u_{\Omega}\right|^{2}-\int_{\Omega} f u_{\Omega}=-\frac{1}{2} \int_{\Omega} f u_{\Omega} .
$$

Proposition 6.1. The shape gradient of the energy functional is given by the expression

$$
d \mathcal{E}(\Omega ; V)=-\frac{1}{2} \int_{\Omega} f u^{\prime}-\int_{\Gamma} f g V \cdot n .
$$

Proof. We have

$$
\mathcal{E}\left(\Omega_{t}\right)=-\frac{1}{2} \int_{\Omega_{t}} f_{t} u_{t}=-\frac{1}{2} \int_{\Omega} f^{t} u^{t} \gamma(t)
$$

and direct calculations show

$$
d \mathcal{E}(\Omega ; V)=-\frac{1}{2} \int_{\Omega} \dot{f} u_{\Omega}+f \dot{u}+f u \operatorname{div} V .
$$


Finally, by an integration by parts, and using the shape derivatives instead of material derivatives, we arrive at the form of the shape gradient of the energy functional given by (6.1). We have taken into account that $f^{\prime}=0$ and $u^{\prime}=\dot{u}-\nabla u \cdot V$.

Let us consider an arbitrary shape functional

$$
J(\Omega)=\frac{1}{2} \int_{\Omega}\left(\frac{u-z}{\varrho}\right)^{2} d x .
$$

Here $z \in W_{0}^{1}\left(\mathbb{R}^{N}\right)$ is a given function.

Proposition 6.2. The shape gradient of functional (6.2) is given by the expression

$$
d J(\Omega ; V)=\int_{\Gamma} \frac{\partial p}{\partial n} \frac{\partial u}{\partial n} V \cdot n d \Gamma(x)+\frac{1}{2} \int_{\Gamma}\left(\frac{u-z}{\varrho}\right)^{2} V \cdot n d \Gamma(x),
$$

where $p$ is given by a solution to the problem

$$
\begin{array}{ll}
-\Delta p=(u-z) \varrho^{-2} & \text { in } \Omega, \\
p=0 & \text { on } \Gamma,
\end{array}
$$

and the data of the problem are sufficiently regular, which means that

$$
\frac{\partial p}{\partial n} \frac{\partial u}{\partial n} \in L_{\mathrm{loc}}^{1}(\Gamma), \quad\left(\frac{u-z}{\varrho}\right)^{2} \in L_{\mathrm{loc}}^{1}(\Gamma) .
$$

Proof. The existence of the shape gradient is obtained by using the material derivatives. Then with the shape derivatives the following expression is derived:

$$
d J(\Omega ; V)=\int_{\Omega}\left(\frac{u-z}{\varrho^{2}}\right) u^{\prime} d x+\frac{1}{2} \int_{\Gamma}\left(\frac{u-z}{\varrho}\right)^{2} V \cdot n d \Gamma(x) .
$$

By an application of Green's formula it follows that

$$
\begin{aligned}
\int_{\Omega}\left(\frac{u-z}{\varrho^{2}}\right) u^{\prime} d x & =-\int_{\Omega} \Delta p u^{\prime} d x=\int_{\Omega} \nabla p \cdot \nabla u^{\prime} d x-\int_{\Gamma} \frac{\partial p}{\partial n} u^{\prime} d \Gamma(x) \\
& =\int_{\Gamma} \frac{\partial p}{\partial n} \frac{\partial u}{\partial n} V \cdot n d \Gamma(x) .
\end{aligned}
$$

The above integral exists under the regularity assumption $\frac{\partial p}{\partial n} \frac{\partial u}{\partial n} \in L_{\mathrm{loc}}^{1}(\Gamma)$ which is satisfied for $u \in W_{1}^{2}\left(\mathbb{R}_{+}^{N}\right)$. Otherwise, for a differentiable shape functional by an application of the structure theorem [2] the shape gradient is given by a distribution compactly supported on the boundary $\Gamma$. 
Acknowledgments. The authors would like to acknowledge the support from Barrande project between the Czech Republic and France. The second author was also supported by the Grant Agency of the Czech Republic, no. 201/02/0684.

\section{References}

[1] C. Amrouche, V. Girault and J. Giroire, Weighted Sobolev spaces for Laplace's equation in $\mathbb{R}^{N}$, J. Math. Pures Appl. 73 (1994), 579-606.

[2] C. Amrouche and Š. Nečasová, Laplace equation in the half-space with a nonhomogeneous Dirichlet boundary condition, Math. Bohemica 126 (2001), 265-274.

[3] T. Z. Boulmezaoud, Espaces de Sobolev avec poids pour l'équation de Laplace dans le demi-espace, C. R. Acad. Sci. Paris Sér. I 328 (1999), 221-226.

[4] M. Delfour and J.-P. Zolésio, Shapes and Geometries: Analysis, Differential Calculus, and Optimisation, Advances in Design and Control, SIAM, Philadelphia, 2001.

[5] B. Hanouzet, Espaces de Sobolev avec poids. Application au problème de Dirichlet dans un demi-espace, Rend. Sem. Univ. Padova 46 (1971), 227-272.

[6] V. G. Maz'ya, B. A. Plamenevskiĭ and L. I. Stupyalis, The three-dimensional problem of steady-state motion of a fluid with a free surface, in: Amer. Math. Soc. Transl. (2) 123 (1984), 171-268.

[7] V. G. Maz'ya and T. O. Shaposhnikova, Multipliers in Spaces of Differentiables Functions, Leningrad Univ., Leningrad, 1986 (in Russian).

[8] J. Sokołowski and J.-P. Zolésio, Introduction to Shape Optimization, Springer, 1992.

Cherif Amrouche

Laboratoire de Mathématiques Appliquées, I.P.R.A.

Université de Pau et des Pays de L'Adour

Av. de l'Université

64000 Pau, France

E-mail: amrouche@smtp.univ-pau.fr

Jan Sokołowski

Institut Elie Cartan

Laboratoire de Mathématiques

Université Henri Poincaré Nancy I

B.P. 239

54506 Vandœuvre-lès-Nancy Cedex, France

E-mail: jan.sokolowski@iecn.u-nancy.fr
Šárka Nečasová Mathematical Institute of the Academy of Sciences

Žitná 25

11567 Praha 1, Czech Republic

E-mail: matus@math.cas.cz 